\title{
Optimization Design of a Multilayer Structure for Broadband and Direction-Selective Emissivity
}

\author{
Cunhai Wang,,$^{1, *}$ Xiaohu Wu, ${ }^{2}$ Fuqiang Wang ${ }^{3}$ and Xinxin Zhang ${ }^{1}$
}

\begin{abstract}
Micro-nano structures for broadband and direction-selective emission have long been a scientific and engineering challenge. In this work, based on the emissivity distribution of three silver-coated dielectric media, including silicon dioxide $\left(\mathrm{SiO}_{2}\right)$, silicon monoxide ( $\mathrm{SiO}$ ), and aluminum oxide $\left(\mathrm{Al}_{2} \mathrm{O}_{3}\right)$, a multilayer structure was designed for broadband and direction-selective emissivity. The structure's emissivity varying with wavelength and emission direction was analyzed. Effects of different arrangement modes of the dielectric media and the thickness of each dielectric layer on the multilayer structure's emissivity were examined for design optimization. The optimal multilayer structure exhibits a strong direction-selective emissivity within a broadband wavelength of 8.0-11.0 $\mu \mathrm{m}$ which covers the spectral emission peak of an object maintained at $\sim 300 \mathrm{~K}$, and thus has potential applications in infrared camouflage.
\end{abstract}

Keywords: Broadband radiation characteristic; Direction-selective emissivity; Multilayer structure; Infrared camouflage.

Received: 15 November 2020; Accepted: 6 February 2021.

Article type: Research article.

\section{Introduction}

According to the Stefan-Boltzmann law, any object at a temperature higher than $0 \mathrm{~K}$ radiates energy in the form of electromagnetic waves. Thus, thermal radiation is a ubiquitous phenomenon and now witnesses essential applications in several domains, such as infrared imaging ${ }^{[1]}$ remote-sensing detection $^{[2]}$, and energy conversion and utilization. ${ }^{[3-8]}$

In general, natural objects are isotropic, and the outwardemitted thermal radiation shows no pronounced directivity. On the other hand, with the development of science and technology, many application fields have set a higher requirement on the direction selectivity of the thermal radiation, and simultaneously, thermal structures with direction selectivity have now attracted a significant amount of attention. Early in 1967, Tien et al. ${ }^{[9,10]}$ from the University of California, Berkeley, discovered the micro-scale radiation effect of the thermal impact on actual engineering. Afterward, investigations on the manipulation of radiation characteristic via micro-structures began to raise broad concerns. In recent years, tremendous micro-/nano- structures, including

\footnotetext{
${ }^{1}$ School of Energy and Environmental Engineering, University of Science and Technology Beijing, Beijing 100083, China.

${ }^{2}$ Shandong Institute of Advanced Technology, Jinan 250100, China

${ }^{3}$ School of New Energy, Harbin Institute of Technology at Weihai,

Weihai 264209, China.

*E-mail: wangcunhai@ustb.edu.cn (C. H. Wang)
}

multilayer slabs, ${ }^{[11-15]} \quad$ nanoparticle/nanowire-doped coatings, ${ }^{[16-20]}$ micro-nano humps, holes and rods, ${ }^{[21-25]}$ and their combined photonic crystals, ${ }^{[26-30]}$ have been prepared for spectral-selective emission of a thermal surface.

In addition to spectral regulation of the radiation emission, controlling the angular emission via sub-wavelength structure is also of great importance in scenarios where radiation in unwanted directions should be suppressed, such as privacy screens, stealth coatings, radar-like detectors. Compared with the fast development of spectral-selective thermal emitters, micro-/nano- designs towards achieving direction-selective thermal emission have been far less prepared. Greffet $e t$ al. ${ }^{[31,32]}$ found that some planar sources have an in-plane spectral coherence length much larger than a wavelength and can be quasi-monochromatic in the near-field. Wolf et al. ${ }^{[33,34]}$ first predicted the spatial coherence emission of a carefully designed microstructure. The work of Greffet et al. ${ }^{\left[{ }^{31,32]}\right.}$ and Wolf et al. ${ }^{[33,34]}$ paves the way for the construction of a thermal source that could radiate light within narrow angular lobes. Greffet et al. ${ }^{[35]}$ prepared silicon carbide (SiC) periodic optical gratings and they observed strong direction-selective emissivity in the wavelength at around $\lambda=11.0 \mu \mathrm{m}$. The highly directional coherent thermal emission therein ${ }^{[35]}$ is due to the diffraction of surface phonon polaritons which can convert surface waves to propagating waves. ${ }^{[35-37]}$ Based on surface plasmons, Laroche et al. ${ }^{[38]}$ and Dahan et al., ${ }^{[39]}$ respectively, designed one-dimensional periodic grating 
(a)

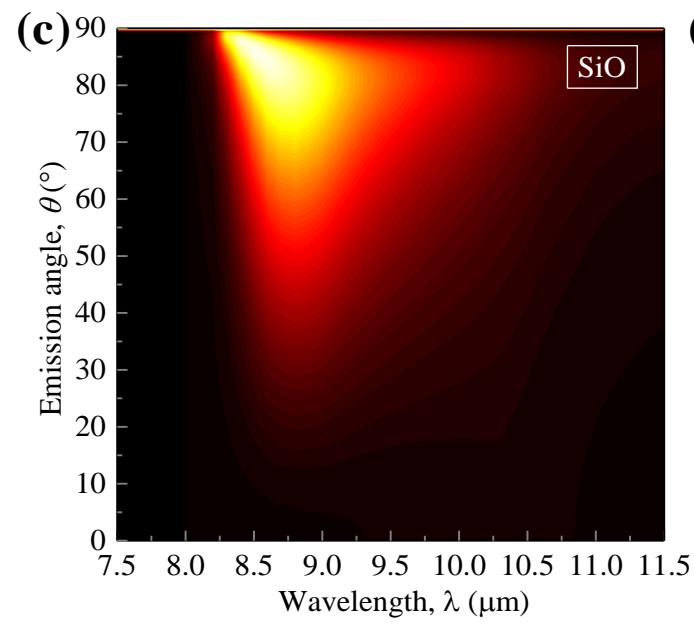

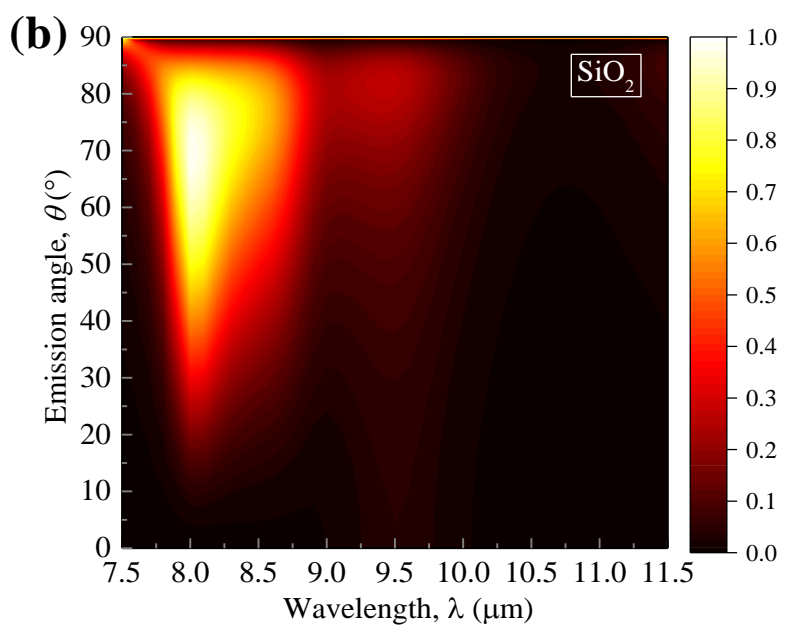

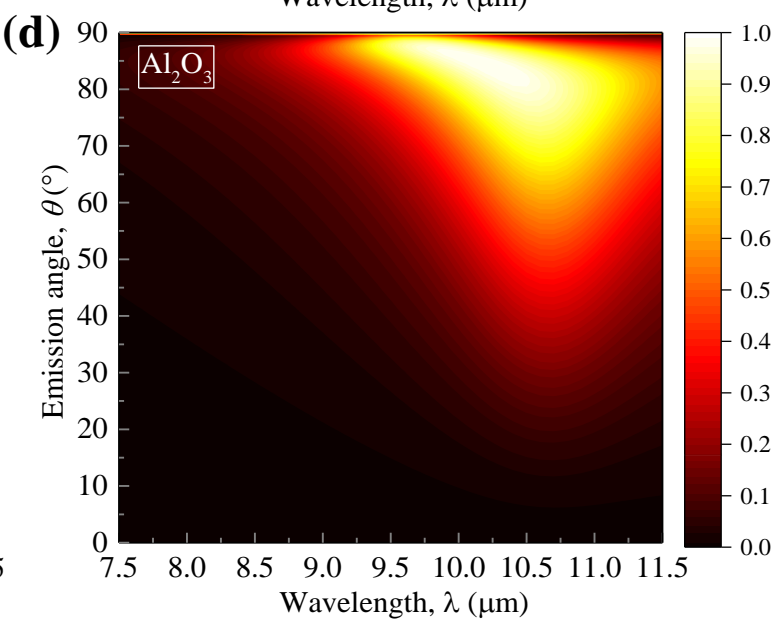

Fig. 1 (a) Sketch of the sDM structure. Emissivity distributions of the sDM structure composed of a $0.2 \mu$ m-thick dielectric medium of (b) $\mathrm{SiO}_{2}$, (c) $\mathrm{SiO}$, and (d) $\mathrm{Al}_{2} \mathrm{O}_{3}$.

structures by using tungsten and silicon dioxide, and achieved intensive direction selectivity in the infrared range. A twodimensional grating structure with both direction selectivity and spectral selectivity was designed by Costantini et al. ${ }^{[40]}$ for applications in infrared light source and gas detection systems. Recent developments on direction-selective emitters can be found in the comprehensive review work on thermal radiation control by Baranov et al. ${ }^{[36]}$ and Wang et al., ${ }^{[37]}$ respectively.

Planck's law demonstrates that thermal radiation emitted from an object at a specific temperature naturally distributes in a broad waveband. Investigating the structure with direction-selective emissivity in a wide wave range is of great relevance to energy conversion and management. ${ }^{[41-45]} \mathrm{Wu}$ and $\mathrm{Fu}[46,47]$ designed a multilayer structure for broadband absorption by carefully stacking the tilted hexagonal boron nitride slabs $^{[46]}$ and silicon nanowire doped polymethyl pentene slabs, ${ }^{[47]}$ respectively. By preparing a two-port system with a graphene layer deposited on a two-dimensional photonic crystal slab, Xiao et al. ${ }^{[48]}$ achieved a manipulation of maximum absorption bandwidth from ultra-narrowband (< $0.5 \mathrm{~nm}$ ) to broadband ( $>50 \mathrm{~nm}$ ). Despite all efforts, the abovereported micro-nano structures can only achieve the direction selectivity of the emissivity at a particular wavelength or in a quite narrow waveband, or broadband emissivity without angular selectivity. Structures with direction-selective emissivity in a broad waveband are far from perfect, ${ }^{[49-51]}$ the existing structures are usually complicated and the optimal designs have been rarely reported.

Thin films composed of epsilon near zero (ENZ) material ${ }^{[52]}$ can couple the electromagnetic modes to the propagation free-space modes, and have been recently verified to have the ability of exhibiting direction-selective emissivity. ${ }^{[53-57]}$ But the previous work is still limited to narrow-band. The objective of this study is to fill the gap of simple structure for obtaining direction-selective emissivity within a broad wave range that covers the wavelength of $\lambda \sim 9.6 \mu \mathrm{m}$ which is the emission peak of a blackbody maintained at the room temperature of $\sim 300 \mathrm{~K}$, by using low-cost ENZ materials.

By carefully checking the optical constant of common oxides, ${ }^{[58]}$ we found that for the materials of $\mathrm{SiO}_{2}, \mathrm{SiO}$, and $\mathrm{Al}_{2} \mathrm{O}_{3}$, the real part $n$ and the imaginary part $k$ of the refractive index satisfies $n \approx k<1.0$ and supports near-zero epsilon phenomenon at specific wavelength $(8.0,8.5,11.0 \mu \mathrm{m}$ for $\mathrm{SiO}_{2}, \mathrm{SiO}$, and $\mathrm{Al}_{2} \mathrm{O}_{3}$, respectively) near $9.6 \mu \mathrm{m}$. We, therefore, spontaneously select these three oxides as the dielectric media for the broadband and direction-selective emitters. The rest of this article is organized as follows. The 
emissivity distributions of a silver-coated dielectric medium are analyzed in Section 2. In Section 3, a multilayer structure composed of three dielectric media was designed for broadband direction-selective emissivity, and optimized for a better performance. Section 4 summarized the main conclusions of this work.

\section{Single-dielectric-medium structure}

Firstly, the emissivity characteristics of the slab structure with a single dielectric medium were investigated. As shown in Fig. 1 (a), a silver ( $\mathrm{Ag}$ ) layer with a thickness of $0.1 \mu \mathrm{m}$ was first deposited on the silicon substrate, and a dielectric medium with a thickness of $d$ was then coated onto the Ag layer. The rigorous coupled wave analysis (RCWA) method, ${ }^{[59-61]}$ which is based on semi-analytical solutions of electromagnetic fields and has been well developed for calculating the emissivity/absorptivity of photonic crystals, ${ }^{[62-65]}$ is applied to calculate the emissivity of the single-dielectric-medium (sDM) structure in this work. Figs. 1(b)-(d) plot the emissivity as a function of wavelength and direction of the sDM structure
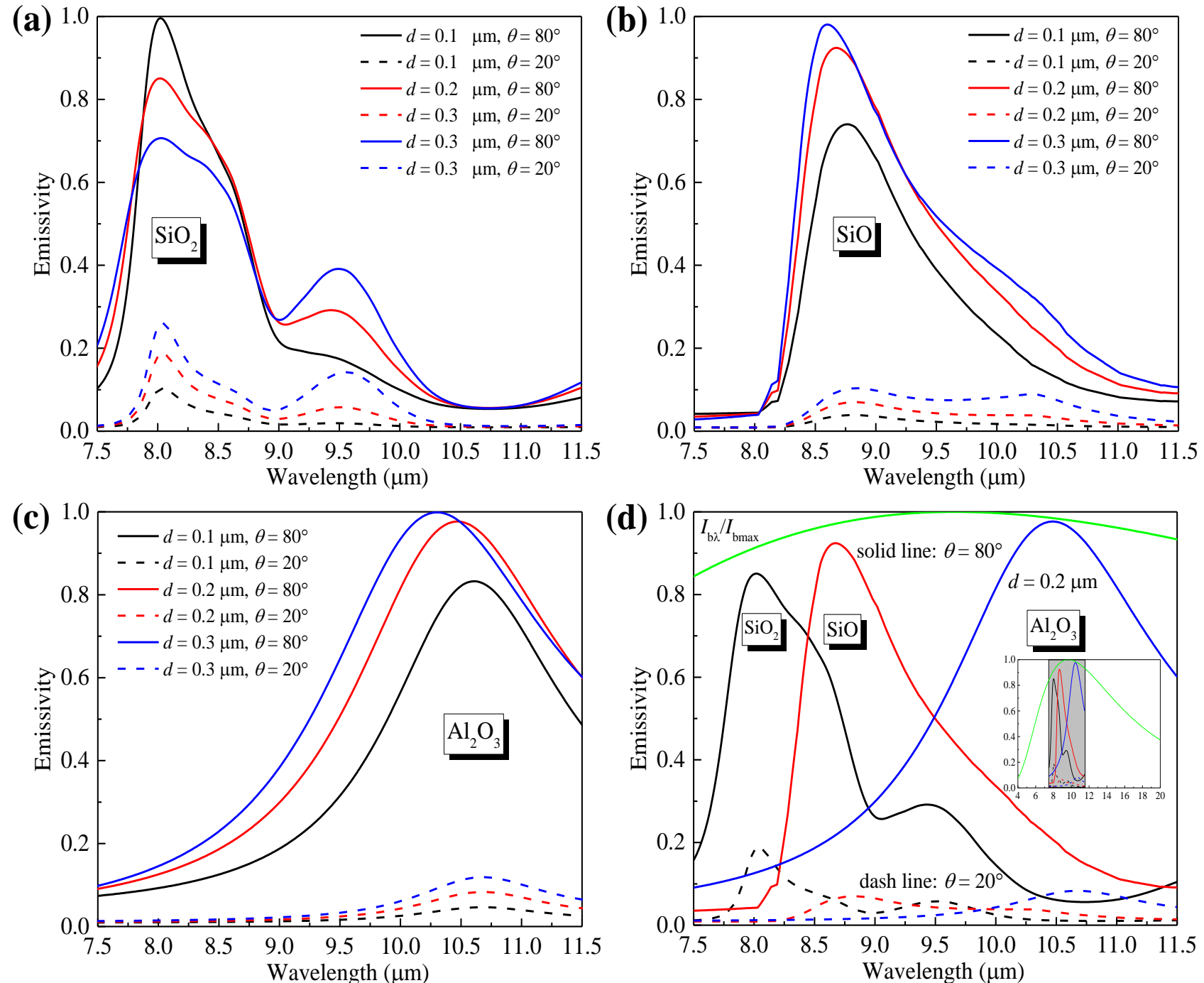

Fig. 2 Emissivities of the sDM structures composed of different dielectric media: (a) $\mathrm{SiO}_{2}$, (b) $\mathrm{SiO}$, and (c) $\mathrm{Al}_{2} \mathrm{O}_{3}$. (d) Emissivity of the different sDM structures composed of a $0.2 \mu \mathrm{m}$-thick dielectric medium and standardized spectral emission intensity of the black body at $300 \mathrm{~K}, I_{\mathrm{b}} \lambda / I_{\mathrm{bmax}}$, where $I_{\mathrm{b} \lambda}$ and $I_{\mathrm{bmax}}$ are the spectral emission intensity and its maximum, respectively. 
It is observed from Figs. 2(a)-(c) that the emissivity differs greatly in the two directions, and the difference is tightly correlated with the thickness of the dielectric layer. When the thickness of the $\mathrm{SiO}_{2}$ dielectric layer is $0.1 \mu \mathrm{m}$, the emissivity peak of the sDM slab reaches a maximum at $80^{\circ}$ and, simultaneously, a minimum at $20^{\circ}$. That is to say, the emissivity difference in these two directions is the greatest when the thickness of the dielectric layer is designed as $d=$ $0.1 \mu \mathrm{m}$. As the thickness of $\mathrm{SiO}_{2}$ increases to $0.2 \mu \mathrm{m}$ or 0.3 $\mu \mathrm{m}$, the emissivity peak in the direction of $80^{\circ}$ drops while the peak in the direction of $20^{\circ}$ rises. In other words, the difference of the emissivity along the two directions drops, suggesting a weakening of the direction selectivity performance. For the structure in which the dielectric layer is composed of $\mathrm{SiO}$ or $\mathrm{Al}_{2} \mathrm{O}_{3}$, the peak emissivities in the two directions increase steadily with the increase of the dielectric thickness, as shown in Figs. 2(b) and 2(c). For the sDM structures composed of the same thickness $d=0.2 \mu \mathrm{m}$ but different dielectric media, the spectral emissivities are plotted together in Fig. 2(d) for the two selected directions. The standardized emission intensity $I_{\mathrm{b}} / I_{\text {bmax }}$ of a blackbody maintained at $300 \mathrm{~K}$ is also plotted in the main body and inset of Fig. 2(d). Here, $I_{\mathrm{b}}$ denotes the spectral emission intensity of a blackbody determined by Planck's law, and $I_{\mathrm{bmax}}$ denotes the maximum of the blackbody spectral emission intensity. Together with the spectral distribution of the standardized blackbody emission, it is noted in Fig. 2(d) that the peak wavelengths of these three structures exhibit a cross-distribution within the wave range covering the peak wavelength of the blackbody emission. This provides a theoretical reference for designing broadband directionalselective emitters at room temperature.

\section{Three-dielectric-medium structure}

In this section, by selecting $\mathrm{SiO}_{2}, \mathrm{SiO}$, and $\mathrm{Al}_{2} \mathrm{O}_{3}$ as the dielectric materials, a multilayer structure with three dielectric

(a)

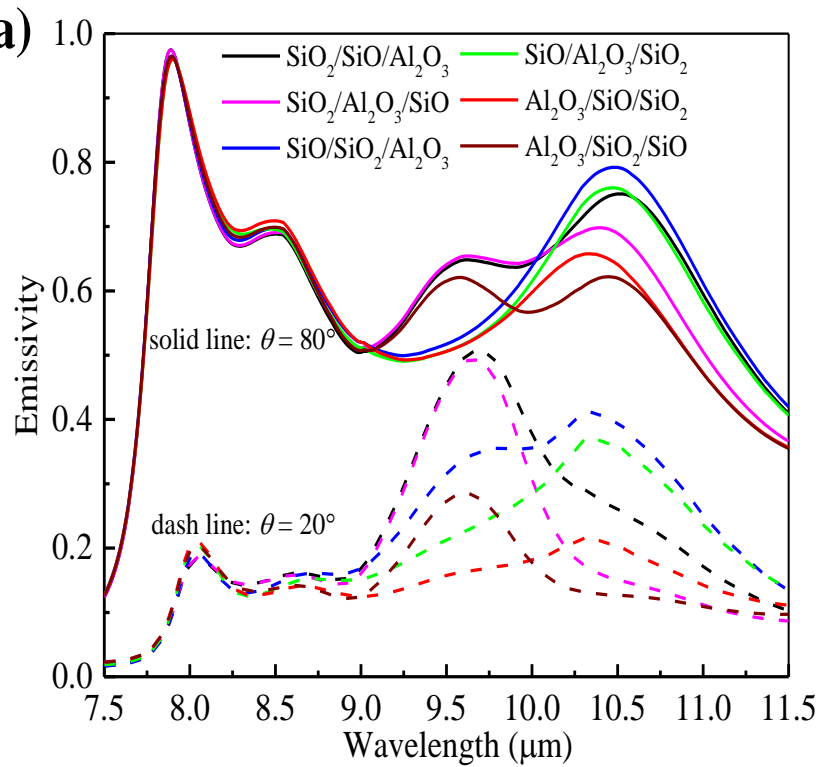

layers was designed. The multilayer is sketched in Fig. 3 and referred to as three-dielectric-medium (tDM) structure in the following. Due to the impedance mismatch, i.e., the dielectric constant difference between the adjacent dielectric media, different arrangements of the three dielectric media have vital influence on the emissivity of the tDM. Therefore, the arrangements of the three dielectric media are first optimized for the desired performance of broadband direction-selective emissivity.

The spectral emissivities of the tDM structure with six different arrangements are shown in Fig. 4(a) where the thickness of each dielectric layer is fixed at $0.2 \mu \mathrm{m}$. A significant emissivity difference is observed among these structures at $\theta=20^{\circ}$ and $\theta=80^{\circ}$. In order to quantitatively compare the direction-selective performance of different tDM structures, the relative emissivity difference along the two selected directions is defined as

$$
\delta=\frac{\varepsilon\left(\theta=80^{\circ}\right)-\varepsilon\left(\theta=20^{\circ}\right)}{\varepsilon\left(\theta=80^{\circ}\right)} X 100 \%
$$

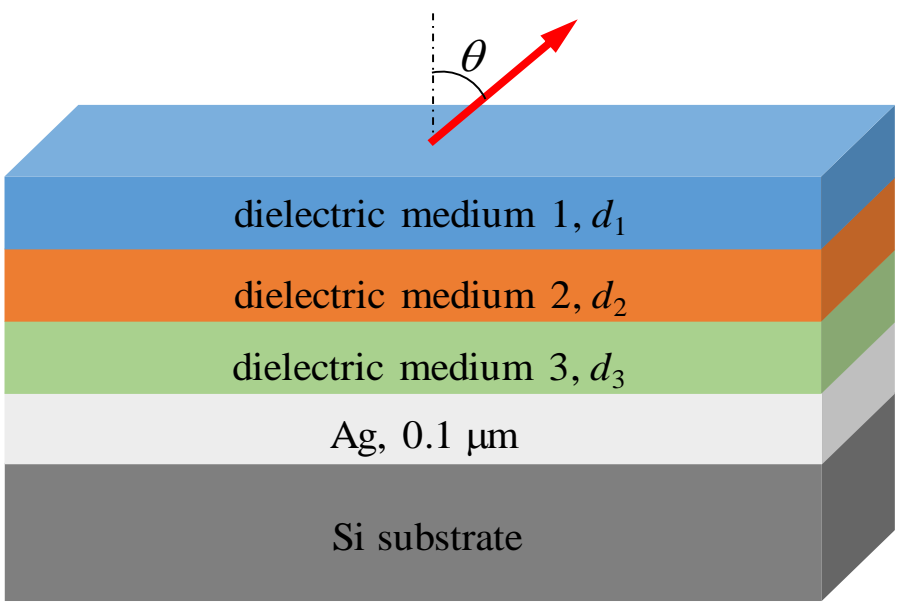

Fig. 3 Sketch of the three-dielectric-medium structure.

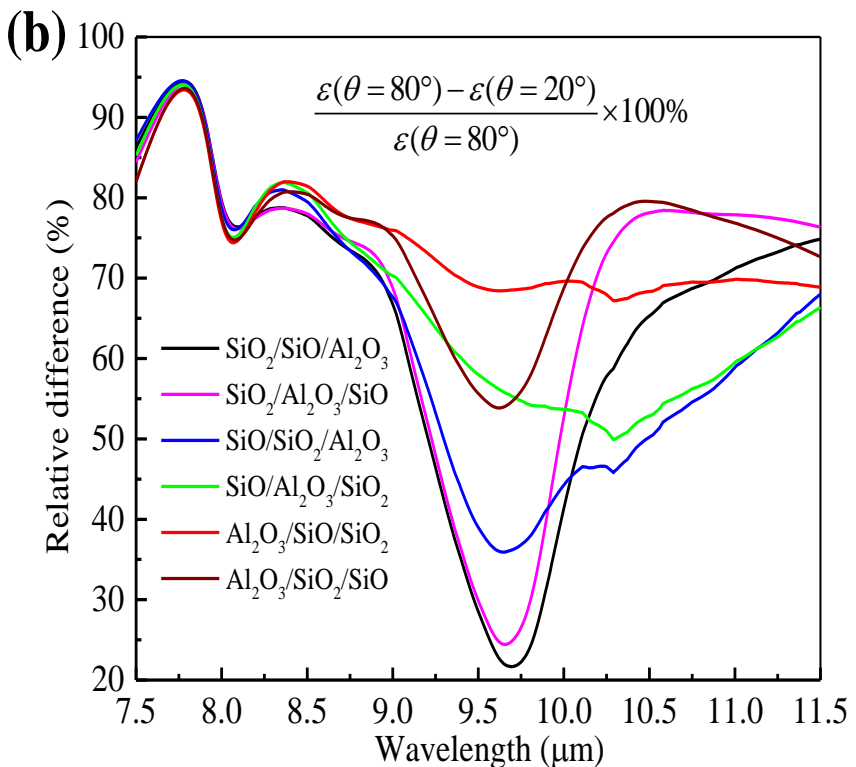

Fig. 4 (a) Emissivities and (b) relative emissivity difference of the tDM structures with different dielectric medium arrangements (the order of the dielectric media listed in each legend entry is from top to bottom). 
(a)

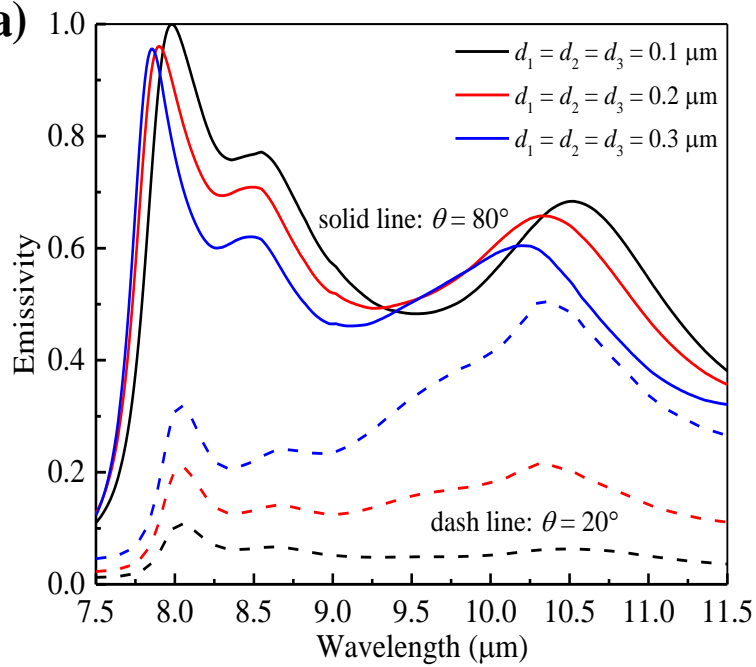

(b)

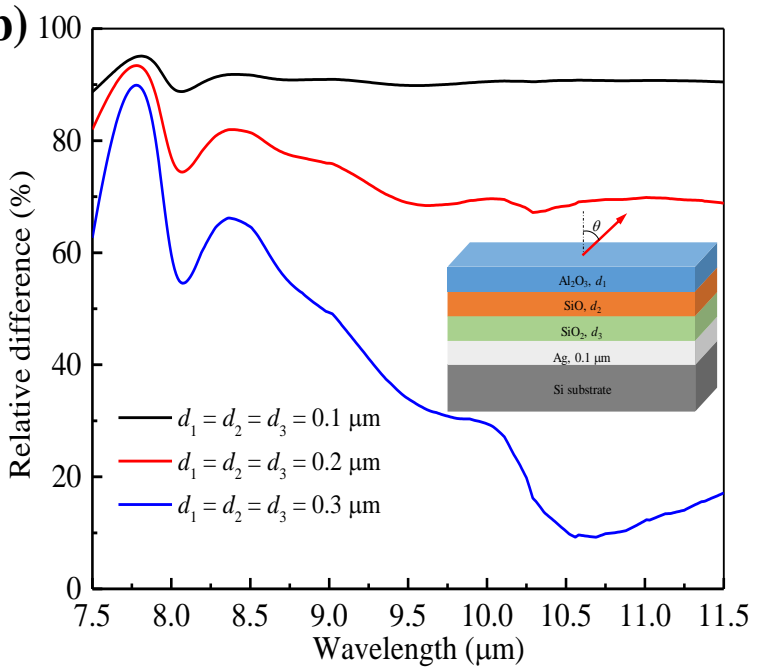

Fig. 5 (a) Emissivities and (b) relative emissivity difference of the tDM structures with equal-thickness dielectric layers.

The emissivity differences of the different structures were compared in Fig. 4(b). When the dielectric medium is composed, from top to bottom, of $\mathrm{Al}_{2} \mathrm{O}_{3}, \mathrm{SiO}$, and $\mathrm{SiO}_{2}$ layers, the relative difference of emissivity along the two directions exceeds $65 \%$, suggesting a good direction selectivity of the emissivity within the wavelength range of $\lambda=7.5-11.5 \mu \mathrm{m}$. The emissivity difference for $\mathrm{tDM}$ structures with other dielectric medium arrangements is found to be lower in some wavelength ranges. Therefore, in order to design tDM structures with an evident direction selectivity of the emissivity in a wide waveband, the dielectric media composing the structure are designed as $\mathrm{Al}_{2} \mathrm{O}_{3}, \mathrm{SiO}$, and $\mathrm{SiO}_{2}$ from top to bottom, the $\mathrm{SiO}$ film between the $\mathrm{Al}_{2} \mathrm{O}_{3}$ and $\mathrm{SiO}_{2}$ films is separated from the air environment to avoid its oxidation. In the following, this tDM structure with $\mathrm{Al}_{2} \mathrm{O}_{3}$, $\mathrm{SiO}$, and $\mathrm{SiO}_{2}$ films from top to bottom is referred to as $\mathrm{Al}_{2} \mathrm{O}_{3}$ $\mathrm{SiO}-\mathrm{SiO}_{2}$-based (ASSb) structure.

In the following, effects of the dielectric thickness on the ASSb structure's emissivity are examined. The scenarios where all dielectric layers have the same thickness were analyzed first. Fig. 5(a) compares the emissivity distributions of the designed structures with three selected dielectric layer

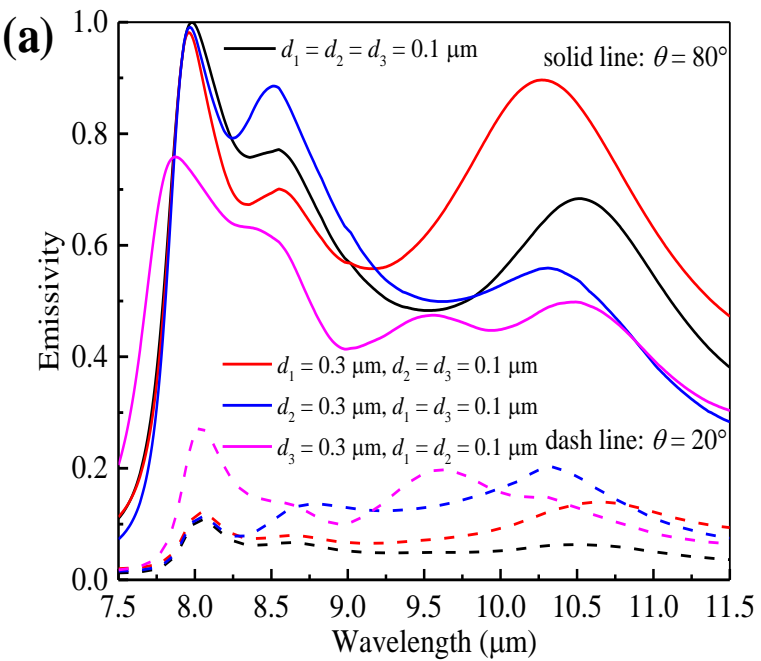

thicknesses of $d=0.1,0.2$, and $0.3 \mu \mathrm{m}\left(d=d_{1}=d_{2}=d_{3}\right.$, where $d_{1}, d_{2}$, and $d_{3}$ are the thicknesses of $\mathrm{Al}_{2} \mathrm{O}_{3}, \mathrm{SiO}$, and $\mathrm{SiO}_{2}$, respectively). It is found that as the thickness increases, the ASSb structure's emissivity at $\theta=20^{\circ}$ increases significantly while the emissivity at $\theta=80^{\circ}$ drops at most wavebands. Therefore, when all layers have the same thickness, the direction selectivity performance of the ASSb structure's emissivity drops with increasing the dielectric layer thickness, as shown in Fig. 5(b).

It is noted from Fig. 2 that the emissivity at $\theta=80^{\circ}$ increases with the dielectric layer thickness for the sDM structure with $\mathrm{Al}_{2} \mathrm{O}_{3}$ or $\mathrm{SiO}$ as the dielectric layer. Therefore, the effects of the dielectric layer thickness on the ASSb structure's emissivity are further examined for the scenarios where the dielectric layers have different thicknesses. Fig. 6(a) displays the emissivities of the structure containing three dielectric layers where two layers remain at $0.1 \mu \mathrm{m}$ and the left layer is increased to $0.3 \mu \mathrm{m}$ thick. In contrast with the results of the case whereby $d_{1}=d_{2}=d_{3}=0.1 \mu \mathrm{m}$, the emissivity at $\theta=20^{\circ}$ is found to increase, no matter which one of the dielectric layers is increased to $0.3 \mu \mathrm{m}$.

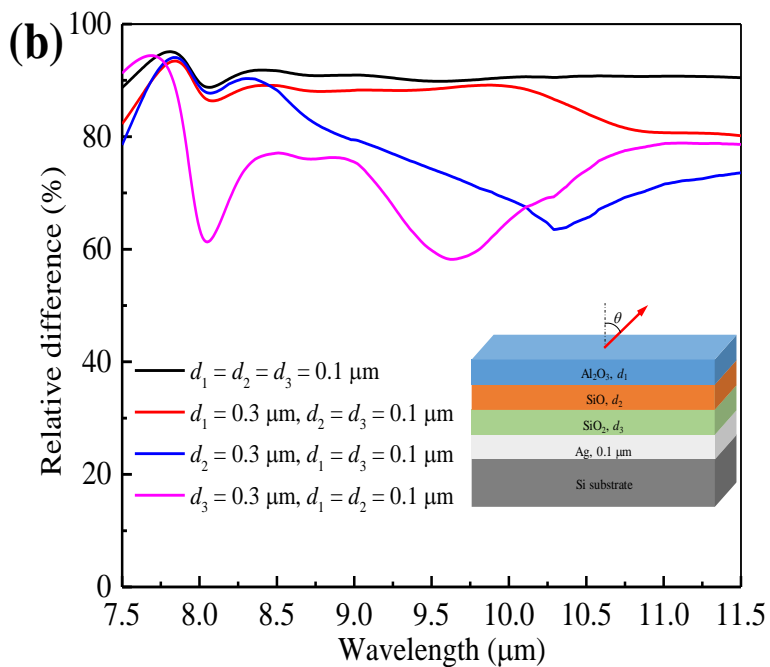

Fig. 6 (a) Emissivities and (b) relative emissivity difference of the tDM structures with different-thickness dielectric layers. 

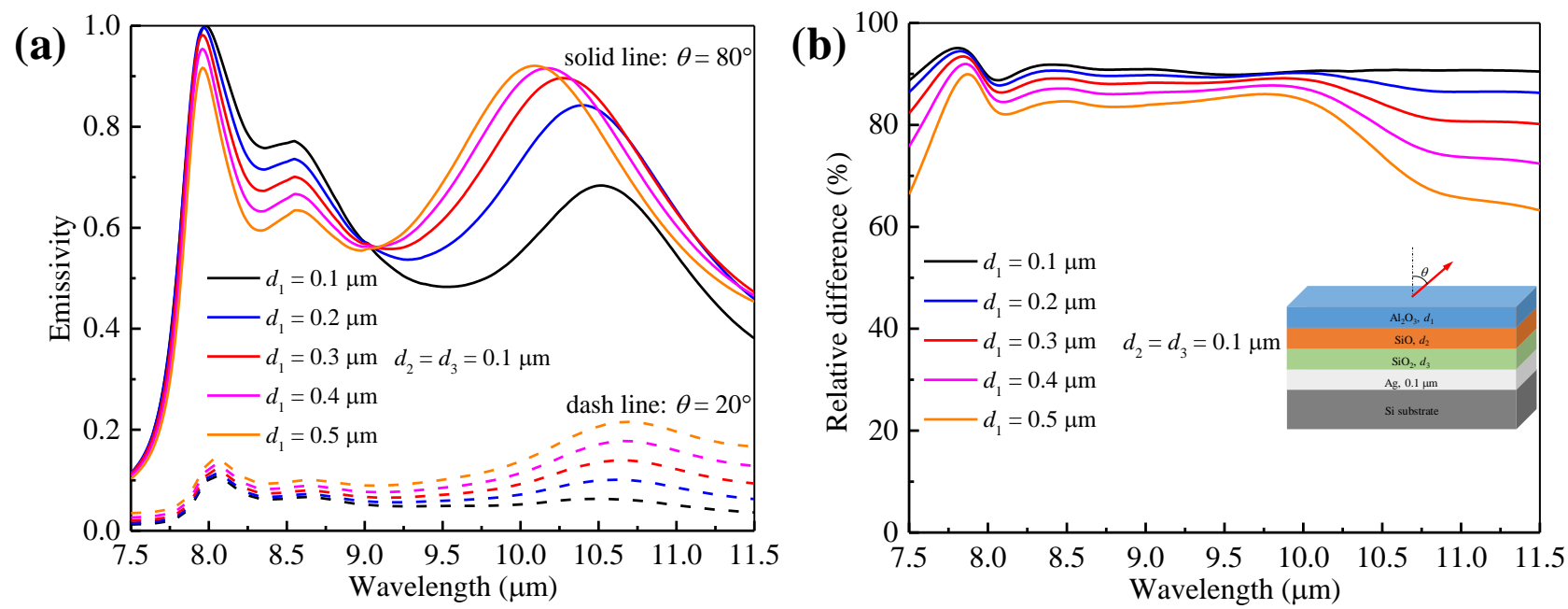

Fig. 7 (a) Emissivities of the slabs composed of multiple dielectric layers with a different-thickness first layer at $\theta=20^{\circ}$ and $80^{\circ}$, and (b) relative difference of the emissivity in the two directions.

When the thickness of the $\mathrm{SiO}_{2}$ dielectric layer increases, the ASSb structure's emissivity at $\theta=80^{\circ}$ increases slightly in the waveband of $\lambda=7.5 \sim 7.8 \mu \mathrm{m}$ while drops pronouncedly in other wavebands. Similarly, when the thickness of the $\mathrm{SiO}$ layer increases, the emissivity at $\theta=80^{\circ}$ increases slightly in the waveband of $\lambda=8.2 \sim 9.7 \mu \mathrm{m}$ while drops significantly in other wavebands. Regarding the emissivity at $\theta=80^{\circ}$ of the structure where the thickness of the $\mathrm{Al}_{2} \mathrm{O}_{3}$ layer is increased to $d_{1}=0.3 \mu \mathrm{m}$, despite the slight decrease in the waveband of $\lambda$ $=7.5 \sim 9.0 \mu \mathrm{m}$, a dramatic increase is found in the waveband of $\lambda=9.5 \sim 11.0 \mu \mathrm{m}$, especially at the wavelength of $\lambda=$ 10.0 10.8 $\mu \mathrm{m}$, as shown in Fig. 6(a).

Fig. 6(b) compares the emissivity difference of the ASSb structures with different layer thicknesses. It can be concluded that the emissivity of the structure maintains a favorable direction selectivity when the thickness of the $\mathrm{Al}_{2} \mathrm{O}_{3}$ layer is set as $d_{1}=0.3 \mu \mathrm{m}$. While the direction-selective performance of the tDM structure is distinctly suppressed if the thickness of the $\mathrm{SiO}_{2}$ or $\mathrm{SiO}$ dielectric layer is increased to $0.3 \mu \mathrm{m}$.

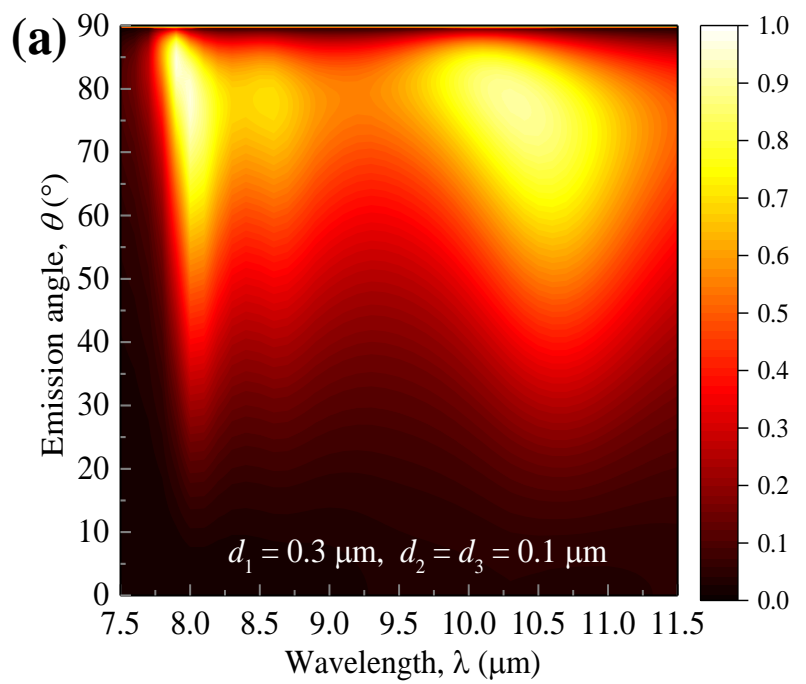

To further study the influence of the $\mathrm{Al}_{2} \mathrm{O}_{3}$ thickness on the emissivity of the ASSb structure, Fig. 7(a) compares the emissivities of the ASSb structures with different $\mathrm{Al}_{2} \mathrm{O}_{3}$ thicknesses $d_{1}=0.1,0.2,0.3,0.4,0.5 \mu \mathrm{m}$, under the condition where the thicknesses of the $\mathrm{SiO}$ and $\mathrm{SiO}_{2}$ remains unchanged as $d_{2}=d_{3}=0.1 \mu \mathrm{m}$. At the low zenith angle of $\theta=20^{\circ}$, the emissivity of the ASSb structure increases steadily as the thickness of the $\mathrm{Al}_{2} \mathrm{O}_{3}$ dielectric layer increases from $0.1 \mu \mathrm{m}$ to $0.5 \mu \mathrm{m}$. At the high zenith angle of $\theta=80^{\circ}$, the emissivity drops in the waveband of $\lambda=7.5 \sim 9.0 \mu \mathrm{m}$, but increases in the waveband of $\lambda=9.5 \sim 10.5 \mu \mathrm{m}$ with increasing the $\mathrm{Al}_{2} \mathrm{O}_{3}$ thickness. Fig. 7(b) plots the difference of the spectral emissivity at the two directions for the ASSb structures with different $\mathrm{Al}_{2} \mathrm{O}_{3}$ layer thicknesses. It can be seen that, as the thickness of the $\mathrm{Al}_{2} \mathrm{O}_{3}$ dielectric layer increases, the $\mathrm{ASSb}$ structure shows a performance loss of the direction-selective emissivity. By conjunctively considering the emissivity in Fig. 7(a) and the emissivity difference in Fig. 7(b), it can be concluded that, when the thickness of the top $\mathrm{Al}_{2} \mathrm{O}_{3}$ layer

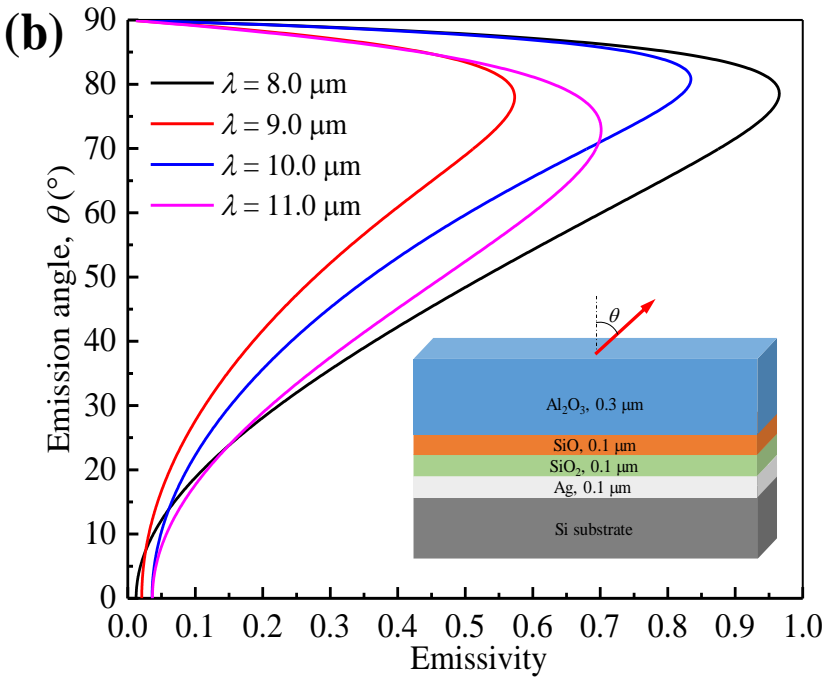

Fig. 8 The optimal ASSb structure's emissivity (a) as a function of wavelength and zenith angle, (b) along four selected wavelengths $\lambda=8.0,9.0,10.0$, and $11.0 \mu \mathrm{m}$ as a function of the emission zenith angle. 
increases from $0.1 \mu \mathrm{m}$ to $0.3 \mu \mathrm{m}$, the spectral emissivity along the direction of $\theta=80^{\circ}$ increases significantly, and the emissivity difference along the two directions maintains higher than $80 \%$. Results in Fig. 7 suggest that the ASSb structure with a $0.3 \mu$ m-thick $\mathrm{Al}_{2} \mathrm{O}_{3}$ dielectric layer shows good emission power and good performance of directionselective emissivity. Thus, the ASSb structure with a $0.3 \mu \mathrm{m}-$ thick $\mathrm{Al}_{2} \mathrm{O}_{3}, 0.1 \mu \mathrm{m}$-thick $\mathrm{SiO}$, and $0.1 \mu \mathrm{m}$-thick $\mathrm{SiO}_{2}$, was chosen as the optimal structure in this work.

The emissivity of the optimal ASSb structure as a function of wavelength and zenith angle is shown in Fig. 8(a). The emissivities at four selected wavelengths $\lambda=8.0,9.0,10.0$, and $11.0 \mu \mathrm{m}$, are plotted in Fig. 8(b) as a function of the emission zenith angle. From the results in Fig. 8, it is seen that the designed structure exhibits a remarkable direction selectivity in the broad wave range of $\lambda=8.0 \sim 11.0 \mu \mathrm{m}$ : the emissivity is high within the zenith angle of $\theta=75^{\circ} \sim 85^{\circ}$, while relatively low within the zenith range of $\theta=0^{\circ} \sim 30^{\circ}$.

The work presented in this paper presents a general strategy for designing and optimizing the performance of a multilayer film for broadband and direction-selective emitter. The high emissivity of the optimal ASSb structure in broadband wavelength enables substantial radiation energy dissipating, and the direction selectivity can suppress radiation emission to the near-normal directions of the surface. Thus, The optimal ASSb structure in this work has potential applications in infrared camouflage and stealth.

\section{Conclusions}

Based on the narrow-band direction-selective emissivity of the silver-coated dielectric medium of $\mathrm{SiO}_{2}, \mathrm{SiO}$, and $\mathrm{Al}_{2} \mathrm{O}_{3}$, a multilayer structure composed of three dielectric media was designed and optimized for broadband direction-selective emissivity. The optimal structure shows a remarkable performance of direction-selective emissivity within a broad wave range of $\lambda=8.0 \sim 11.0 \mu \mathrm{m}$ which covers the emission peak of a blackbody at $\sim 300 \mathrm{~K}$. The emissivity is high at a large zenith angle while low at a small zenith angle. The emissivity difference at two directions $\left(\theta=20^{\circ}\right.$ and $\left.80^{\circ}\right)$ of the optimal ASSb structure is higher than $80 \%$ in a broad waveband. The designed multilayer structure can significantly reduce infrared emission in the direction perpendicular to the interface while maintaining sustainable outward radiation energy, thereby exhibits important application in many prospects, such as infrared camouflage and stealth.

\section{Acknowledgements}

This work was supported by National Natural Science Foundation of China (No. 51906014), Natural Science Foundation of Shandong Province (No. ZR2020LLZ004), and the Fundamental Research Funds for the Central Universities (No. FRF-BD-20-09A).

\section{Supporting information}

Not applicable

\section{Conflict of interest}

There are no conflicts to declare.

\section{References}

[1] H Zuo, D Y Choi, X Gai, P Ma, L Xu, D N Neshev, B Zhang, B Luther-Davies, Adv. Opt. Mater, 2017, 5, 1700585, doi: 10.1002/adom.201700585

[2] G Yan, Z H Jiao, T Wang, X Mu, Remote Sens. Environ., 2020, 237, 111556. doi: 10.1016/j.rse.2019.111556.

[3] F Wang, L Ma, Z Cheng, J Tan, X Huang, L Liu, Renew. Sust. Energ. Rev., 2017, 73, 935-949, doi: 10.1016/j.rser.2017.01.165.

[4] Y Gao, Z Wang, D Ding, W Li, Y Ma, Y Hao, H Zhang, ES Energy Environ., 2019, 5, 1-7, doi: 10.30919/esee8c328.

[5] M Chen, Y He, Sol. Energy. Mater. Sol. Cells, 2018, 188, 156-163. doi: 10.1016/j.solmat.2018.09.003.

[6] W Li, S Fan, Opt. Express, 2018, 26, 15995-16021, doi: 10.1364/OE.26.015995.

[7] H Xu, H H Chen, S Wang, Z Li, K Li, G Stecker, W Pan, J J Lee, C L Chen, Int. J. Heat Mass Transf., 2017, 107, 468-483, doi: 10.1016/j.ijheatmasstransfer.2016.11.061.

[8] S Fan, Joule, 2017, 1, 264-273, doi: 10.1016/j.joule.2017.07.012.

[9] C L Tien, Adv. Heat Transf., 1969, 5, 253-324, doi: 10.1016/S0065-2717(08)70131-X.

[10] E G Cravalho, C L Tien, R P Caren, J. Heat Transf., 1967, 89, 351-358, doi: 10.1115/1.3614396.

[11] A P Raman, M A Anoma, L Zhu, E Rephaeli, S Fan, Nature, 2014, 515, 540-544, doi: 10.1038/nature13883.

[12] P Yang, C Chen, Z M Zhang, Sol. Energy, 2018, 169, 316324. doi: 10.1016/j.solener.2018.04.031.

[13] Y Shi, W Li, A Raman, S Fan, ACS Photonics, 2017, 5, $684-$ 691. doi: 10.1021/acsphotonics.7b01136.

[14] M Hu, G Pei, Q Wang, J Li, Y Wang, J Ji, Appl. Energy, 2016, 179, 899-908, doi: 10.1016/j.apenergy.2016.07.066.

[15] W Li, Y Shi, K Chen, L Zhu, S Fan, ACS Photonics, 2017, 4, 774-782, doi: 10.1021/acsphotonics.7b00089.

[16] A R Gentle, G B Smith, Nano Lett., 2010, 10, 373-379, doi: $10.1021 / \mathrm{nl} 903271 \mathrm{~d}$.

[17] H Bao, C Yan, B Wang, X Fang, C Y Zhao, X Ruan, Sol. Energy Mater. Sol. Cells, 2017, 168, 78-84. doi: 10.1016/j.solmat.2017.04.020.

[18]Z Huang, X Ruan, Int. J. Heat Mass Transf., 2017, 104, 890896. doi: 10.1016/j.ijheatmasstransfer.2016.08.009.

[19]Z Cheng, F Wang, D Gong, H Liang, Y Shuai, Sol. Energy Mater. Sol. Cells, 2020, 213, 110563, doi: 10.1016/j.solmat.2020.110563.

[20] T Li, Y Gao, K Zheng, Y Ma, D Ding, H Zhang, ES Energy Environ., 2019, 5, 102-107, doi: 10.30919/esee8c325.

[21] C Zou, G Ren, M M Hossain, S Nirantar, W Withayachumnankul, T Ahmed, M Bhaskaran, S Sriram, M Gu, C Fumeaux, Adv. Opt. Mater., 2017, 5, 1700460, doi: 10.1002/adom.201700460.

[22] J Song, J Seo, J Han, J Lee, B J Lee, Appl. Phys. Lett., 2020, 117, 094101, doi: 10.1063/5.0017838.

[23]Z Yu, N P Sergeant, T Skauli, G Zhang, H Wang, S Fan, Nat. Commun., 2013, 4, 1-7, doi: 10.1038/ncomms2765. 
[24] L Zhu, A P Raman, S Fan, Proc. Natl. Acad. Sci., 2015, 112, 12282-12287, doi: 10.1073/pnas.1509453112.

[25] M A Baqir, A Farmani, M Razas, M N Akhtar, A Hussain, Appl. Nanosci., 2021, 11, 229-240, doi: 10.1007/s13204-02001574-w.

[26] B Zhao, M Hu, X Ao, Q Xuan, G Pei, Sol. Energy Mater. Sol. Cells, 2018, 178, 266-272, doi: 10.1016/j.solmat.2018.01.023.

[27] E Rephaeli, A Raman, S Fan, Nano Lett., 2013, 13, 1457 1461. doi: $10.1021 / \mathrm{nl} 4004283$.

[28] X Wang, J Duan, W Chen, C Zhou, Y Liu, S Xiao, Phys. Rev. $B$, 2020, 102, 155432, doi: 10.1103/PhysRevB.102.155432.

[29] M J He, H Qi, Y T Ren, Y J Zhao, M Antezza, Int. J. Heat Mass Transf., 2020, 150, 119305, doi: 10.1016/j.ijheatmasstransfer.2020.119305.

[30]H Liang, X Zhang, B Lin, F Wang, Z Cheng, X Shi, B G Lougou, ES Energy Environ., 2020, 10, 22-33, doi: 10.30919/esee8c728.

[31] R Carminati, J J Greffet, Phys. Rev. Lett., 1999, 82, 16601663, doi: 10.1103/PhysRevLett.82.1660.

[32] A V Shchegrov, K Joulain, R Carminati, J J Greffet, Phys. Rev. Lett., 2000, 85, 1548-1551, doi: 10.1103/physrevlett.85.1548. [33] E Wolf, Nature, 1987, 326, 363-365. doi: 10.1038/326363a0. [34] E Wolf, D F James. Correlation-induced spectral changes. Rep. Prog. Phys., 1996, 59, 771-818, doi: 10.1088/00344885/59/6/002.

[35] J J Greffet, R Carminati, K Joulain, J P Mulet, S Mainguy, Y Chen Nature, 2002, 416, 61-64, doi: 10.1038/416061a.

[36] D G Baranov, Y Xiao, I A Nechepurenko, A Krasnok, A Alu, M A Kats, Nat. Mater., 2019, 18, 920-930, doi: 10.1038/s41563019-0363-y.

[37] B X Wang, M Q Liu, T C Huang, C Y Zhao, ES Energy Environ., 2019, 6, 18-38, doi: 10.30919/esee8c360.

[38] M Laroche, C Arnold, F Marquier, R Carminati, J J Greffet, S Collin, N Bardou, J L Pelouard, Opt. Lett., 2005, 30, 2623-2625, doi: 10.1364/OL.30.002623.

[39] N Dahan, A Niv, G Biener, V Kleiner, E Hasman, Phys. Rev. B, 2007, 76, 045427, doi: 10.1103/PhysRevB.76.045427.

[40] D Costantini, A Lefebvre, A L Coutrot, I Moldovan-Doyen, J P Hugonin, S Boutami, F Marquier, H Benisty, J J Greffet, Phys. Rev. Appl., 2015, 4, 014023, doi: 10.1103/PhysRevApplied.4.014023.

[41] E D Kosten, J H Atwater, J Parsons, A Polman, H A Atwater, Light-Sci. Appl., 2013, 2, e45, doi: 10.1038/1sa.2013.1.

[42] R Sakakibara, V Stelmakh, W R Chan, M Ghebrebrhan, J D Joannopoulos, M Soljacic, I Celanovic, J. Photonics Energy, 2019, 9, 032713, doi: 10.1117/1.JPE.9.032713.

[43] E Lucchi, Renew. Sust. Energ. Rev., 2018, 82, 3077-3090, doi: 10.1016/j.rser.2017.10.031.

[44] O Hohn, T Kraus, G Bauhuis, U T Schwarz, B Blasi, Opt. Express, 2014, 22, A715-A722, doi: 10.1364/OE.22.00A715.

[45] E Sakr, P Bermel, Opt. Express, 2017, 25, A880-A895, doi: 10.1364/oe.25.00a880.

[46] X Wu, C Fu, J. Quant. Spectrosc. Radiat. Transf., 2018, 209, 150-155. doi: 10.1016/j.jqsrt.2018.01.031.

[47] X Wu, C Fu, Nanosc. Microsc. Therm., 2018, 22, 114-123, doi: 10.1080/15567265.2018.1434844.

[48] S Xiao, T Liu, X Wang, X Liu, C Zhou, Phys. Rev. B, 2020, 102, 085410, doi: 10.1103/PhysRevB.102.085410.

[49] J H Atwater, P Spinelli, E Kosten, J Parsons, C Van Lare, J Van de Groep, J Garcia de Abajo, A Polman, H A Atwater, Appl. Phys. Lett., 2011, 99, 151113, doi: 10.1063/1.3648115.

[50] Y Shen, D Ye, I Celanovic, S G Johnson, J D Joannopoulos, M Soljacic, Science, 2014, 343, 1499-1501, doi: 10.1126/science.1249799.

[51] Y Shen, C W Hsu, Yeng YX, JD Joannopoulos. Broadband angular selectivity of light at the nanoscale: Progress, applications, and outlook. Appl. Phys. Rev., 2016, 3, 011103, doi: 10.1063/1.4941257.

[52] I Liberal, N Engheta, Nat. Photonics, 2017, 11, 149-158. doi: 10.1038/nphoton.2017.13.

[53] L V Alekseyev, E E Narimanov, T Tumkur, H Li, Y A Barnakov, M A Noginov, Appl. Phys. Lett., 2010, 97, 131107, doi: 10.1063/1.3469925.

[54] J Rensberg, Y Zhou, S Richter, C Wan, S Zhang, P Schoppe, R Schmidt-Grund, S Ramanathan, F Capasso, M A Kats, C Ronning, Phys. Rev. Appl., 2017, 8, 014009, doi: 10.1103/PhysRevApplied.8.014009.

[55] T Shaykhutdinov, A Furchner, J Rappich, K Hinrichs, Opt. Mater. Express, 2017, 7, 3706-3714, doi: 10.1364/OME.7.003706.

[56] M A Badsha, Y C Jun, C K Hwangbo, Opt. Commun., 2014, 332, 206-213, doi: 10.1016/j.optcom.2014.07.004.

[57] L J Krayer, J Kim, J L Garrett, J N Munday, ACS Photonics, 2019, 6, 2238-2244, doi: 10.1021/acsphotonics.9b00449.

[58]E D Palik. Handbook of Optical Constants of Solids. Academic Press, San Diego, CA, 1998.

[59] M G Moharam, T K Gaylord, J. Opt. Soc. Am. A, 1981, 71, 811-818, doi: 10.1364/JOSA.71.000811.

[60] M G Moharam, E B Grann, D A Pommet, T K Gaylord, J Opt. Soc. Am. A, 1995, 12, 1068-1076. doi: 10.1364/JOSAA.12.001068.

[61] L Li, J Opt. Soc. Am. A, 1996, 13, 1870-1876, doi: 10.1364/JOSAA.13.001870.

[62] L P Wang, B J Lee, X J Wang, Z M Zhang, Int. J. Heat Mass Transf., 2009, 52, 3024-3031. doi: 10.1016/j.ijheatmasstransfer.2009.01.042.

[63] V Liu, S FanS, Comput. Phys. Commun., 2012, 183, $2233-$ 2244, doi: 10.1016/j.cpc.2012.04.026.

[64] L P Wang, Z M Zhang, Appl. Phys. Lett., 2012, 100, 063902, doi: 10.1063/1.3684874.

[65] E Rephaeli, A Raman, S Fan, Nano Lett., 2013, 13, 14571461. doi: 10.1021/n14004283.

\section{Author information}

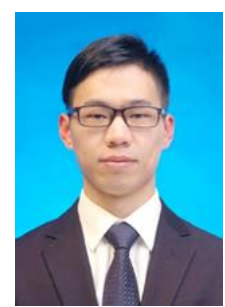

Cun-Hai Wang is an associate professor at University of Science and Technology Beijing. He received his M.S., B.S., and Ph.D. degrees at Harbin Institute of Technology in 2011, 2013, and 2018, respectively, under the guidance of Prof. Hong-Liang Yi. He was a 
visiting student at University of California, Berkeley from Sept. 2015 to Oct. 2016 under the guidance of Prof. Costas. P. Grigoropoulos. As the first or corresponding author, he has published more than 20 SCI-index papers. His scientific research concentrates on multi-scale photothermal radiation transfer, including numerical methods for solving radiative transfer equation in participating media, radiation characteristic of micro-nano structures, radiative cooling, and near-field radiative heat transfer.

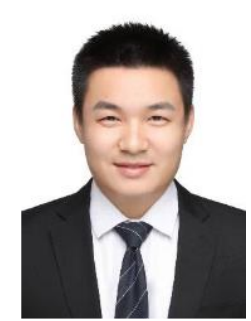

Xiao-Hu is an associate researcher at Shandong Institute of Advanced Technology. He received his B.S. degree in engineering mechanics from China University of Mining and Technology (Beijing) and Ph. D. degree from Peking University under the guidance of Prof. Ceji Fu. He was a visiting student at Georgia Institute of Technology from Sept. 2017 to Sept. 2018 under the guidance of Prof. Zhuomin Zhang. His main research interest is on thermal radiative properties of anisotropic materials and applications. His work about hyperbolic materials was selected as "Optics in 2020" by Optics \& Photonics News.

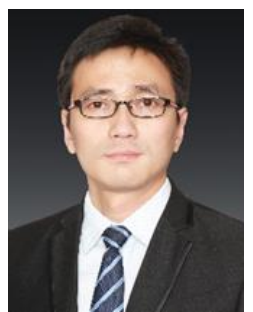

Fu-Qiang Wang is a Professor at Harbin Institute of Technology at Weihai. He has hosted 3 National Natural Science Funds of China, and published 54 SCI -index papers (1st/corresponding author), 10 papers have been selected as ESI papers. Besides, He have won the Natural Science First and Second Prize of Heilongjiang Province, and Taishan Young Scholar of Shandong Province, Doctoral Academic Newcomer Award of the Ministry of Education, Awards of highly cited paper of Applied Energy journal and Outstanding Contribution of Young and Middle-aged Experts of Weihai. He is the director of Weihai Key Laboratory of New Energy. His current research interests include thermal radiation and solar energy conversion and utilization.

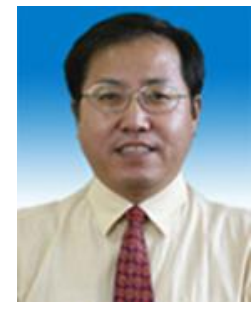

Xin-Xin Zhang is a Professor at University of Science and Technology Beijing. He served as the headmaster of University of Science and Technology Beijing from 2013 to 2018. His research is in the area of energy conversion and management, including thermophysical properties, multiscale heat transfer, clean energy technologies, biothermophysics. He is now the head of the research group of "Heat and Mass Transfer and Thermophysical Measurements" in School of Energy and Environmental Engineering at University of Science and Technology Beijing.

Publishers Note: Engineered Science Publisher remains neutral with regard to jurisdictional claims in published maps and institutional affiliations. 